\title{
Synchrony in tritrophic food chain metacommunities
}

Igor Belykh (ibelykh@gsu.edu)

Carlo Piccardi (carlo.piccardi@polimi.it)

Sergio Rinaldi (rinaldi@elet.polimi.it)

\section{Approved by}

Ulf Dieckmann

Leader, Evolution and Ecology Program

June 2010

Interim Reports on work of the International Institute for Applied Systems Analysis receive only limited review. Views or opinions expressed herein do not necessarily represent those of the Institute, its National Member Organizations, or other organizations supporting the work. 


\section{Contents}

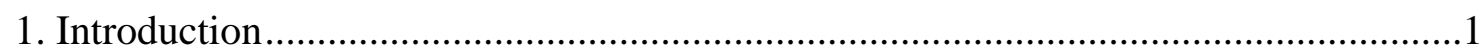

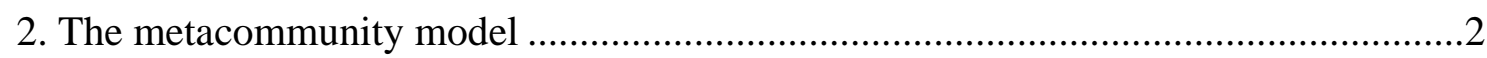

3. Local synchronization .....................................................................................

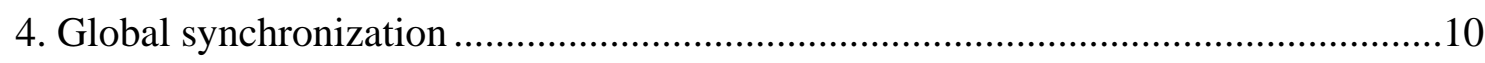

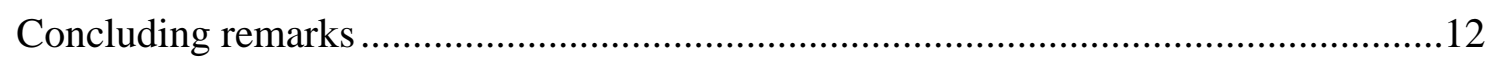

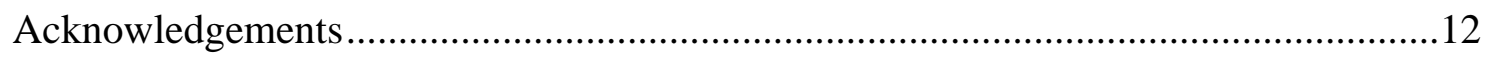

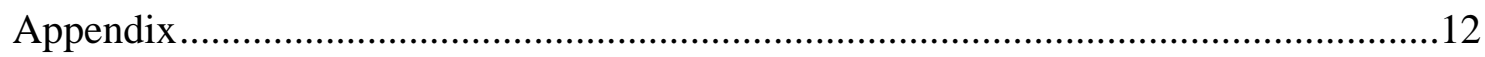

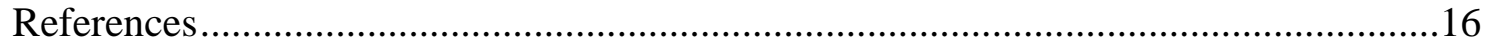




\title{
Synchrony in Tritrophic Food Chain Metacommunities
}

\author{
Igor Belykh ${ }^{\mathrm{a}}$, Carlo Piccardi ${ }^{\mathrm{b}}$, and Sergio Rinaldi ${ }^{\mathrm{b}, \mathrm{c} *}$ \\ a Department of Mathematics and Statistics, Georgia State University, Atlanta, GA, \\ USA; ${ }^{b}$ Dipartimento di Elettronica e Informazione, Politecnico di Milano, Milano, Italy; \\ ${ }^{\mathrm{c}}$ EEP, International Institute for Applied Systems Analysis, Laxenburg, Austria
}

(May 8, 2008)

Keywords: First keyword; Second keyword; from two to five.

AMS Subject Classification: F1.1; F4.3; from two to six AMS 2000 Subject Classification codes.

\section{Introduction}

In the last decades many plant and animal populations have been shown to synchronize (i.e., to behave in unison) over large areas. Examples are aphids and butterflies [21, 30, 53], moths [27, 33, 37], fish [43], crabs [24], birds [12, 44], voles and mice [9, 25], hares [43], squirrels [43, 45], sheep [20], arctic herbivores [41], early blooming forest floor species [40], as well as many others [29, 32]. Synchrony is the consequence of two independent factors: the dispersal of populations among patches and the existence of common meteorological driving forces (Moran effect). The theory is well established: see [10, 26, 38] for dispersal, [48] for the Moran effect, and [15] for the mixed case.

The contribution that first revealed that populations can synchronize over large distances was the study of fur returns of Canadian lynx to the Hudson Bay Company [18]. Since then, a great effort has been devoted to this case study (see $[11,19,36,45,51,52]$, just to mention a few), with some of these contributions supporting the idea that the synchronization of Canadian lynx is primarily due to global environmental fluctuations. However some recent results [10, 34, 35] have shown that networks of tritrophic food chain models of the kind suggested for the food web of the Canadian boreal forest [28], can easily give rise to synchronization in the absence of a common meteorological driving force, provided there is a sufficiently high dispersal. This result and the discovery of a high gene flow of the Canadian lynx over distances of $3000 \mathrm{~km}$ [50] suggest that, most likely, migration is a key factor for the spatial synchronization of the lynx population.

This is why we concentrate in this paper on the case in which dispersal is the only cause of synchrony. Thus, our metacommunity is characterized by three basic

${ }^{*}$ Corresponding author. Mailing address: Prof. Sergio Rinaldi, Dipartimento di Elettronica e Informazione, Politecnico di Milano, Piazza Leonardo Da Vinci 32, I-20133 Milano, Italy; Email: rinaldi@elet.polimi.it 
elements: a graph in which nodes $i$ and $\operatorname{arcs}(i, j)$ represent patches and dispersal flows between patches; a model describing the dynamics of the population vector $n^{(i)}$ in patch $i$ when isolated from the other patches; the dispersal rates of each component of the population vector.

Depending upon the metacommunity model, various forms of synchronization are possible. The strongest one is complete synchronization which occurs when a synchronous solution $n^{(i)}(t)=n^{(j)}(t)$ for all $i \neq j$ and for all $t$ exists and is stable. A slightly weaker form of synchronization, called almost-complete synchronization, requires that the maximum difference between populations is small. Much weaker but quite interesting forms of synchronization are phase synchronization $[10,13$, 34], out-of-phase synchronization [17, 49], and intermittent synchronization [14, 22]. In general, when the parameters of a metacommunity are not too far from the values giving rise to complete synchronization, i.e., when the parameters are in the so-called pre-synchronization region, some weak form of synchronization is present and is revealed by various spatio-temporal patterns.

In this paper, we first show how the available general theory of local synchronization $[1,26,38]$ can be used to discuss networks of food chains composed of resource, consumer and predator populations. The patch model we use is a standard tritrophic food chain model and the main result is that the dispersal of the consumer population is much more effective than those of the other populations in promoting synchronous behaviors. Then, we show how factors like competition, interference, and cooperation among consumers or predators can be taken into account in order to detect if they promote synchronization or if they oppose to it. The results are consistent with a general principle that says that stabilizing factors promote synchronization. Finally, the analysis is extended to the study of global synchronization through the use of the general available theory [4-7].

\section{The metacommunity model}

The metacommunity we consider in this paper is composed of $N$ identical patches connected through corridors, along which migration occurs in both directions. Each patch $i(i=1,2, \ldots, N)$ is occupied by $p$ populations whose abundances are the components of a $p$-dimensional vector $n^{(i)}$. The metacommunity is described by

$$
\dot{n}^{(i)}(t)=f\left(n^{(i)}(t)\right)+d \sum_{j \in S_{i}} H\left(n^{(j)}(t)-n^{(i)}(t)\right), \quad i=1,2, \ldots, N
$$

where $\dot{n}^{(i)}(t)=f\left(n^{(i)}(t)\right)$ is the patch model, namely the equation governing the dynamics of an isolated patch; $S_{i}$ is the set of patches directly connected to patch $i$ (i.e., the neighborhood of $i$ ); $d$ is dispersal; and $H=\operatorname{diag}\left[h_{1}, h_{2}, \ldots, h_{p}\right]$ (with $h_{l} \geq 0$ for all $l$, and $\sum_{l} h_{l}=1$ ) is the dispersion profile, i.e., a constant diagonal matrix specifying the propensity of dispersing of all components of the population vector.

A particular solution of (1), called synchronous solution, is characterized by

$$
n^{(1)}(t)=n^{(2)}(t)=\cdots=n^{(N)}(t) \quad \forall t .
$$

As a matter of fact, when (2) holds, all the dispersal flows $d H\left(n^{(j)}(t)-n^{(i)}(t)\right)$ in (1) vanish for all $t$, and the dynamics of each patch is simply governed by $\dot{n}(t)=f(n(t))$. Notice that this is possible thanks to the assumption that all patches are identical. When (2) holds, the trajectory of system (1) is confined to 


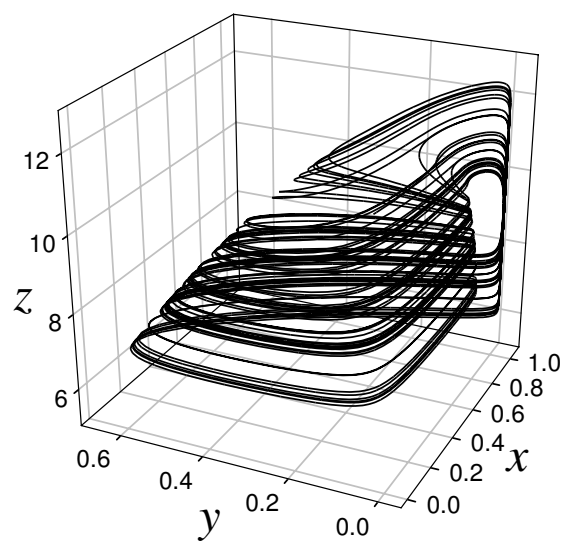

Figure 1. The tea-cup chaotic attractor of the Rosenzweig-McArthur model (3) with parameter values (4).

a $p$-dimensional linear manifold $\Sigma$ called synchronization manifold. It is of utmost importance to assess whether the synchronous solution (2) is stable, i.e., whether system (1) converges to (2) from any nearby initial state (in which case we say that the metapopulation displays local synchronization) or from any state (global synchronization). In the following two sections, we study these two problems with reference to thritrophic food chain metacommunities, where the components $x, y$, and $z$ of the three-dimensional population vector $n$ are the abundances of resource, consumers, and predators in the patch. The dynamics of the three populations in an isolated patch are described by

$$
\begin{aligned}
& \dot{x}=r x\left(1-\frac{x}{K}\right)-\frac{a_{1} x y}{1+a_{1} b_{1} x}, \\
& \dot{y}=e_{1} \frac{a_{1} x y}{1+a_{1} b_{1} x}-m_{1} y-\frac{a_{2} y z}{1+a_{2} b_{2} y}, \\
& \dot{z}=e_{2} \frac{a_{2} y z}{1+a_{2} b_{2} y}-m_{2} z
\end{aligned}
$$

where $r$ and $K$ are net growth rate and carrying capacity of the resource, and $\left(a_{i}, b_{i}, d_{i}, e_{i}\right), i=1,2$, are attack rate, handling time, death rate and efficiency of consumers $(i=1)$ and predators $(i=2)$. Model (3) is known as RosenzweigMacArthur model since it is the natural extension to three trophic levels of the model proposed long ago by Rosenzweig and MacArthur [47] for ditrophic food chains. Model (3) can have chaotic behavior [23] but in relatively narrow ranges of its parameter space [31]. Here the parameters are fixed at the following values (already used in the literature as reference values):

$$
\begin{aligned}
r & =1.15, \quad K=1.07 \\
a_{1} & =5, \quad b_{1}=0.6, \quad m_{1}=0.4, \quad e_{1}=1, \\
a_{2} & =0.1, \quad b_{2}=20, \quad m_{2}=0.0037, \quad e_{2}=1,
\end{aligned}
$$

because for these values the model has a chaotic attractor (Fig. 1) and the discussion of the possibility of synchronizing the metapopulation by suitably selecting the dispersal $d$ is particularly interesting. 


\section{Local synchronization}

Through straightforward manipulations, equation (1) can be rewritten as

$$
\dot{n}^{(i)}(t)=f\left(n^{(i)}(t)\right)-d \sum_{j=1}^{N} g_{i j} H n^{(j)}(t), \quad i=1,2, \ldots, N
$$

where the $N \times N$ connectivity matrix $G=\left[g_{i j}\right]$ describes the topology of the network composed of patches and corridors. More precisely, for $i \neq j, g_{i j}=g_{j i}=$ -1 if patches $i, j$ are directly connected and $g_{i j}=g_{j i}=0$ otherwise, whereas $g_{i i}=-\sum_{j \neq i} g_{i j}$ is the degree of patch $i$, i.e., the number of corridors rooted at $i$. To avoid degeneracies, we assume that any pair of patches is connected either directly or through a chain of corridors. Thus $G$ is a real, symmetric, irreducible matrix. In addition, all off-diagonal elements are non-positive, and each row has zero-sum. As a consequence, the eigenvalues $\lambda_{i}$ of $G$ are real and

$$
0=\lambda_{1}<\lambda_{2} \leq \lambda_{3} \leq \cdots \leq \lambda_{N}
$$

Given a network, i.e., a connectivity matrix $G$, the local stability of the synchronization manifold $\Sigma$ can be ascertained by looking at the evolution of the differences $\left(n^{(i)}(t)-n^{(1)}(t)\right), i=2,3, \ldots, N$, which are described, after neglecting the higherorder terms in the Taylor expansion, by a $p \times(N-1)$-dimensional linear system with time-varying Jacobian matrix given by

$$
J=\left[\begin{array}{ccc}
\frac{\partial f}{\partial n}-d\left(g_{22}-g_{12}\right) H & \cdots & -d\left(g_{2 N}-g_{1 N}\right) H \\
\vdots & \ddots & \vdots \\
-d\left(g_{N 2}-g_{12}\right) H & \cdots & \frac{\partial f}{\partial n}-d\left(g_{N N}-g_{1 N}\right) H
\end{array}\right]
$$

Through a suitable change of coordinates based on the eigenvectors of the matrix $G$, it can be shown $([26,38])$ that this Jacobian matrix is equivalent to a blockdiagonal matrix with matrices $\left[\partial f / \partial n-d \lambda_{i} H\right], i=2,3, \ldots, N$, on the diagonal. Such matrices describe the dynamics of the metapopulation close to the synchrony manifold. Thus, if the largest Liapunov exponents of these matrices, denoted by $L_{\left[\partial f / \partial n-d \lambda_{i} H\right]}$, are negative, i.e.

$$
L_{\left[\partial f / \partial n-d \lambda_{i} H\right]}<0, \quad i=2,3, \ldots, N
$$

then the synchronous solution (2) is locally stable, i.e., the metapopulation synchronizes.

Given a metacommunity, conditions (5) can easily be checked by using any standard algorithm (e.g. [42]) for the computation of the largest Liapunov exponent of each time varying matrix

$$
\frac{\partial f}{\partial n}-\lambda_{i} d H, \quad i=2,3, \ldots, N
$$

where $\partial f / \partial n$ is evaluated along a solution of $\dot{n}=f(n)$. These matrices depend upon the patch characteristics (patch model $f$ and dispersion profile $H$ ) and upon the product $\lambda_{i} d$. Given $f$ and $H$, one can therefore consider the family of matrices

$$
\frac{\partial f}{\partial n}-\varepsilon H
$$


and denote by $L(\varepsilon)$ the largest Liapunov exponent of each element of the family. The function $L(\varepsilon)$ is known as Master Stability Function (MSF) [38], and is very useful for discussing the impact of the patch characteristics on the synchronization of the metapopulation.

In fact, if the isolated patch is chaotic, as model (3)-(4) is, then $L(0)=\bar{L}>0$ (where $\bar{L}$ is the largest Liapunov exponent of the matrix $\partial f / \partial n$ ), so that three types of MSFs can be considered:

(i) $L(\varepsilon)>0$ for all $\varepsilon>0$,

(ii) $L(\varepsilon)<0$ for $\varepsilon>\underline{\varepsilon}>0$,

(iii) all other cases.

Type (i) MSFs guarantee that condition (5) cannot be satisfied, i.e., no metapopulation can synchronize even for high dispersal. By contrast, type (ii) MSFs guarantee that all metapopulations can synchronize provided the dispersal is sufficiently high, i.e.

$$
d>\underline{d}=\frac{1}{\lambda_{2}} \underline{\varepsilon} .
$$

The threshold value $\bar{d}$ depends upon the network $G$ through the eigenvalue $\lambda_{2}$, which is very high $\left(\lambda_{2}=N\right)$ in fully connected networks and very small in weakly connected ones (e.g. $\lambda_{2} \simeq 1 / N^{2}$ in a circular lattice), but $\underline{d}$ also depends upon the patch characteristics $(f, H)$ through $\underline{\varepsilon}$. Finally, type (iii) MSFs guarantee that some (but not all) metapopulations can synchronize if dispersal can be suitably selected. For example, if $(\underline{\varepsilon}, \bar{\varepsilon})$ is an interval where $L(\varepsilon)<0$, then in all networks with

$$
\frac{\lambda_{N}}{\lambda_{2}}<\frac{\bar{\varepsilon}}{\underline{\varepsilon}},
$$

the synchronous solution is stable for

$$
\frac{1}{\lambda_{2}} \underline{\varepsilon}<d<\frac{1}{\lambda_{N}} \bar{\varepsilon} .
$$

i.e., synchrony can be lost if dispersal is increased. It is worth noticing that (7) is always satisfied in fully connected networks, because in that case all positive eigenvalues of $G$ coincide.

The MSF of model (3)-(4) has been computed for the three dispersion profiles

$$
\begin{aligned}
H^{\mathrm{I}} & =\operatorname{diag}[1,0,0], \\
H^{\mathrm{II}} & =\operatorname{diag}[0,1,0], \\
H^{\mathrm{III}} & =\operatorname{diag}[0,0,1],
\end{aligned}
$$

corresponding to the extreme cases in which only one of the three populations disperses, and the result, shown in Fig. 2, is quite interesting since the MSF is of type (i), (ii), and (iii) in cases I, II, and III, respectively. This means that no metacommunity can synchronize if dispersal involves only the resource, while any metacommunity can synchronize if only consumers disperse. No counterexample has been found to these rules which, however, should not be taken as theorems. By contrast, the fact that the MSF is of type (iii) when only predators disperse is not robust because type (ii) MSFs have also been obtained for $H=H^{\mathrm{III}}$ by 


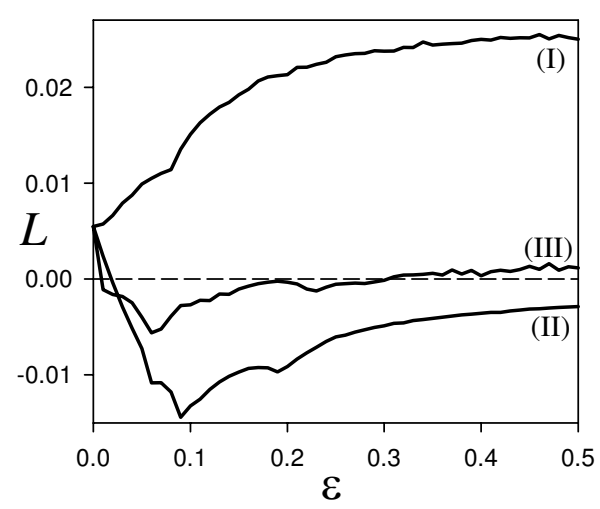

Figure 2. The Master Stability Functions of the Rosenzweig-McArthur model (3)-(4) when the only dispersing species is the resource $\left(H=H^{\mathrm{I}}\right.$, type (i)), the consumers $\left(H=H^{\mathrm{II}}\right.$, type (ii)), or the predators $\left(H=H^{\mathrm{III}}\right.$, type (iii)).

(a)

(b)

Figure 3. The sketch of a type (ii) $\operatorname{MSF} L(\varepsilon, \delta)$ and of the function $\underline{\varepsilon}(\delta)$ : (a) $\delta$ is stabilizing; (b) $\delta$ is destabilizing.

varying the parameters of the patch model. However, the result shown in Fig. 2 is of interest because until now type (iii) MSFs have been detected in very few chaotic oscillators [1].

Let us now show how one can detect the impact of a demographic parameter on synchronization. For this, once the MSF $L(\varepsilon)$ has been computed for a reference model (like model (3) with the reference parameter values (4)), let us indicate with $\delta$ a positive variation of a parameter and recompute the MSF $L(\varepsilon, \delta)$ for the perturbed model. Thus, $L(\varepsilon, 0)$ is the MSF of the reference model, i.e., one of the three functions described in Fig. 2, and $L(0,0)=\bar{L}$.

If the reference MSF $L(\varepsilon, 0)$ is of type (ii) then, by continuity, the $\operatorname{MSF} L(\varepsilon, \delta)$ will also be of type (ii), provided $\delta$ is not too large. In other words, for sufficiently small parameter perturbations there exists a function $\underline{\varepsilon}(\delta)$ such that $L(\underline{\varepsilon}(\delta), \delta)=0$. The functions $L(\varepsilon, \delta)$ and $\underline{\varepsilon}(\delta)$ are sketched in Fig. 3 under the naive assumption that the MSF depends linearly upon $\varepsilon$ and $\delta$. Figure $3(\mathrm{a})$ depicts the case of a so-called stabilizing parameter [46] (i.e., a parameter that reduces the Liapunov exponent $L(0, \delta)$ of a single isolated patch), while Fig. 3(b) depicts the opposite case of a destabilizing parameter $(L(0, \delta)$ increasing with $\delta)$. Figure $3($ a) shows that $\underline{\varepsilon}(\delta)$ decreases with $\delta$, so that the synchronization condition (6) can be satisfied with lower dispersal when $\delta$ increases. For this reason, in the case of Fig. 3(a), if the dispersal is fixed then high values of $\delta$ promote synchronization, i.e., $\delta$ is synchronizing. Conversely, in Fig. 3(b) the function $\underline{\varepsilon}(\delta)$ is increasing and $\delta$ is desynchronizing. The conclusion is that in the case of type (ii) MSFs the synchronizing factors are nothing but the stabilizing factors.

The same conclusion does not hold for MSFs of type (i) and (iii). In fact, if the reference MSF is of type (i), then it remains such for small parameter perturbations $\delta$, so that the synchronization of the metacommunity is not possible no matter if $\delta$ is stabilizing or destabilizing. In the case of type (iii) MSFs, Fig. 3 still makes sense for small values of $\varepsilon$ and $\delta$, so that the minimum dispersal needed for synchronization, 
namely $\underline{\varepsilon}(\delta) / \lambda_{2}$, decreases [increases] with $\delta$ if $\delta$ is stabilizing [destabilizing]. By contrast, no relationship can reasonably be established between stabilizing factors and the loss of synchronization at high dispersal rates, because nothing can be inferred on $\bar{\varepsilon}(\delta)$. However, understanding what happens at high dispersal rates is not particularly relevant in the context of metacommunities because it is known that many (if not all) populations are characterized by very low dispersal rates.

The results we have just pointed out are very important for various reasons. First, because they allow one to infer a property of a metacommunity (synchronization) from a property of a single community (stabilizing or destabilizing demographic parameter). This implies that the impact of a demographic factor on the emergence of collective dynamics (like synchronization) can reasonably be conjectured on the basis of observations or experiments performed on a single patch. Second, the results reinforce a recent analogous conclusion [15], namely that biological chaos is an obstacle for the synchronization of metacommunities through Moran effect. In other words, the idea that stabilizing [destabilizing] factors are synchronizing [desynchronizing] seems to be valid in general and not only for the case examined in this paper dealing with synchronization due exclusively to dispersal. Finally, the bridge we have established between local (i.e., patch) chaos and global (i.e., network) synchronization reinforces an important evolutionary conjecture [16], namely that biological evolution drives local dynamics toward the edge of chaos and global dynamics toward weak forms of synchronization.

Obviously, the conclusions drawn from Fig. 3, which depicts a naive and simplified interpretation of a MSF, cannot be argued to hold in general, even if the same conclusions can obviously be obtained (through the implicit function theorem) under a slightly more general assumption, namely that $L(\varepsilon, \delta)$ is decreasing with respect to $\varepsilon$ and decreasing [increasing] with respect to $\delta$ when $\delta$ is stabilizing [destabilizing]. Thus, it makes sense to check whether the results derived from Fig. 3 hold for particular classes of metacommunities and this is, indeed, what we do in the following for our class of tritrophic food chains.

Instead of studying the effects on synchronization of the parameters appearing in the patch model (3), we now try to see if some demoghraphic phenomena which are not taken into account in the Rosenzweig-MacArthur model (3) are synchronizing or desynchronizing. More precisely, we consider six phenomena, namely competition, interference, and cooperation among consumers or predators, and we measure with a small positive parameter $\delta$ the relevance of the phenomen. Obviously, for $\delta=0$ we obtain model (3) where, indeed, the six phenomena are neglected. For $\delta \neq 0$, one or two equations of model (3) must be modified as specified below.

1. Intraspecific competition among consumers: In agreement with the derivation of the classical logistic equation $\dot{x}=r x(1-x / K)$ (see resource equation in the absence of consumers), the intraspecific competition among consumers can be dealt with by introducing an extra-mortality proportional to $y^{2}$ in the consumer equation, that becomes

$$
\dot{y}=e_{1} \frac{a_{1} x y}{1+a_{1} b_{1} x}-m_{1} y-\frac{a_{2} y z}{1+a_{2} b_{2} y}-\delta y^{2} .
$$

2. Intraspecific competition among predators: For consistency with the previous case, we must only perturb the predator equation, that becomes:

$$
\dot{z}=e_{2} \frac{a_{2} y z}{1+a_{2} b_{2} y}-m_{2} z-\delta z^{2} .
$$

3. Interference among consumers: Interference among individuals feed- 
ing on a common resource is usually taken into account by lowering the functional response of the individuals as first proposed in [2]. Thus, interference among consumers requires to modify the first two equations of model (3) as follows:

$$
\begin{aligned}
& \dot{x}=r x\left(1-\frac{x}{K}\right)-\frac{a_{1} x y}{1+a_{1} b_{1} x+\delta y}, \\
& \dot{y}=e_{1} \frac{a_{1} x y}{1+a_{1} b_{1} x+\delta y}-m_{1} y-\frac{a_{2} y z}{1+a_{2} b_{2} y} .
\end{aligned}
$$

4. Interference among predators: For consistency with the previous case, we must modify the last two equations of model (3) in the following way:

$$
\begin{aligned}
& \dot{y}=e_{1} \frac{a_{1} x y}{1+a_{1} b_{1} x}-m_{1} y-\frac{a_{2} y z}{1+a_{2} b_{2} y+\delta z}, \\
& \dot{z}=e_{2} \frac{a_{2} y z}{1+a_{2} b_{2} y+\delta z}-m_{2} z .
\end{aligned}
$$

5. Cooperation among consumers: If cooperation enhances predation, we can simply multiply the consumer functional response by a factor greater than 1 , which is here written as $\left(1+\delta y /\left(y+y_{0}\right)\right)$, where the parameter $y_{0}$ is fixed ( $y_{0}=0.3$ in our numerical analysis). This means that abundant consumers predate $(1+\delta)$ times more than scarce consumers. Thus, the first two equations of model (3) must be modified as follows:

$$
\begin{aligned}
& \dot{x}=r x\left(1-\frac{x}{K}\right)-\frac{a_{1} x y}{1+a_{1} b_{1} x}\left(1+\delta \frac{y}{y+y_{0}}\right), \\
& \dot{y}=e_{1} \frac{a_{1} x y}{1+a_{1} b_{1} x}\left(1+\delta \frac{y}{y+y_{0}}\right)-m_{1} y-\frac{a_{2} y z}{1+a_{2} b_{2} y} .
\end{aligned}
$$

6. Cooperation among predators: Similarly to the previous case, the modified consumer and predator equations are $\left(z_{0}=9\right.$ in our numerical analysis):

$$
\begin{aligned}
& \dot{y}=e_{1} \frac{a_{1} x y}{1+a_{1} b_{1} x}-m_{1} y-\frac{a_{2} y z}{1+a_{2} b_{2} y}\left(1+\delta \frac{z}{z+z_{0}}\right), \\
& \dot{z}=e_{2} \frac{a_{2} y z}{1+a_{2} b_{2} y}\left(1+\delta \frac{z}{z+z_{0}}\right)-m_{2} z .
\end{aligned}
$$

In order to make the remaining part of this section more attractive, this is a good point for conjecturing, on a purely intuitive background, which factors are synchronizing and which are desynchronizing. For doing this, one can use the idea previously pointed out, i.e., stabilizing [destabilizing] factors are synchronizing [desynchronizing]. Thus, for example, one might imagine that stronger predators can be more effective in keeping their prey under control, thus avoiding large fluctuations of the consumer population. This means that one should be inclined to imagine that factor 6 , namely cooperation among predators, should be a stabilizing factor and, hence, also a synchronizing factor. Before proceeding, the reader is invited to make his/her own guess for each of the six above factors.

In order to detect the impact of all factors on synchronization, we have systematically computed the largest Liapunov exponent $L(\varepsilon, \delta)$ on a $25 \times 25$ grid for each one of the 6 factors and for $H=H^{\mathrm{II}}$ (dispersing consumers) as well as for 


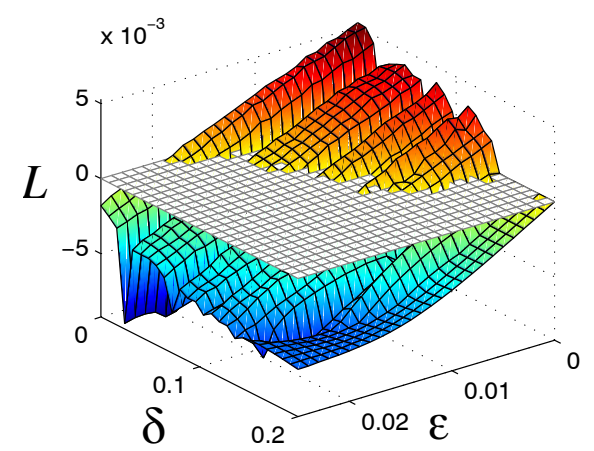

Figure 4. The MSF for the case of interference among dispersing consumers $\left(H=H^{\mathrm{II}}\right.$, factor 3$)$.

Figure 5. The MSF, for low values of $\varepsilon$ and $\delta$, for the case of intraspecific competition among consumers when predators disperse $\left(H=H^{\mathrm{III}}\right.$, factor 1$)$.

Figure 6. The MSF for the case of cooperation among dispersing consumers $\left(H=H^{\mathrm{II}}\right.$, factor 5$)$.

$H=H^{\mathrm{III}}$ (dispersing predators). Thus, our analysis has required the computation of $2 \times 6 \times 25 \times 25=7500$ largest Liapunov exponents of a $3 \times 3$ time-varying Jacobian matrix, that have been computed with a standard algorithm [42]. Obviously, in none of the examined cases the MSF $L(\varepsilon, \delta)$ is linear in $\varepsilon$ and $\delta$ (as in Fig. 3), neither it is monotone (recall that, in chaotic regions, there are always thin subregions of regular behavior where the largest Liapunov exponent drops to zero). However, the statement "stabilizing [destabilizing] factors are synchronizing [desynchronizing] factors" turns out to be true in all cases. The result of the analysis is that four factors, namely $1,3,4$, and 6 , are stabilizing and hence synchronizing, while the remaining two, namely 2 and 5 , are destabilizing and hence desynchronizing, no matter which is the dispersing population. Figure 4 shows one example of the first class, namely the case of interference among dispersing consumers: the MSF is of type (ii) and the two functions $L(0, \delta)$ and $\underline{\varepsilon}(\delta)$ qualitatively recall those reported in Fig. 3(a) even if they are not monotonically decreasing. However, it is fully justified to summarize Fig. 4 by saying that consumer interference is a synchronizing factor. Similarly, Fig. 5 shows the case of intraspecific competition among consumers with dispersing predators. Again the functions $L(0, \delta)$ and $\underline{\varepsilon}(\delta)$ resemble only vaguely to those of Fig. 3(a) but still the result is that intraspecif competition among consumers is a synchronizing factor. Finally, an example of a desynchronizing factor is shown in Fig. 6. 


\section{Global synchronization}

In this section, we show how the general results on the global stability of synchronization can be applied to the metacommunity (1)-(3).

Most methods for the global stability of synchronized limit-cycle or chaotic systems are based on the calculation of the eigenvalues of the connectivity matrix for coupling topologies and a term depending mainly on the dynamics of the individual oscillators $[3,8,39,54,55]$. These studies show that the global stability of synchronization depends on the eigenvalues of the Laplacian connection matrix.

An alternate way to establish synchrony which does not depend on explicit knowledge of the spectrum of the connection matrix was developed in [4]. This Connection Graph Method combines the Lyapunov function approach with graph theoretical reasoning. In this context the main step is to establish a bound on the total length of all paths passing through an edge on the network connection graph.

The method guarantees complete synchronization from arbitrary initial conditions and not just local stability of the synchronization solution. The coupling strength may vary from pair to pair of interacting cells and it may even depend on time.

In the following, we review the method and derive the synchronization conditions for networks (1)-(3) with dispersal profiles admitting global synchronization.

It is worth noticing that once global synchronization is settled at the critical value of coupling, a further increased dispersal (even up to an infinite value) cannot desynchronize the regime of global synchrony (the proof follows from the Lyapunov function discussed in Appendix; further details can be found in [4]). This is in contrast with local synchronization which can appear in a certain interval of coupling parameters and become unstable with coupling increasing (cf. Case III where only predators disperse). Therefore, the Master Stability function calculated for the stability of local synchronization in the previous section (cf. Fig. 2) allows us to single out dispersal profile $H^{\mathrm{II}}$ (Case II) where one can expect to observe global synchronization. In addition, we also relate our analysis to the most general profile (not discussed in the previous section) where $H^{0}=\operatorname{diag}\left[h_{1}, h_{2}, h_{3}\right]$ and $h_{1}, h_{2}$, and $h_{3}$ may range from 0 to 1 . Here, all three trophic components of the food chain (resource, consumers, and predators) can equally disperse.

The method states that the coupling threshold required for the global stability of synchronization in a network with any arbitrary configurations can be predicted from the threshold for global synchronization in the simplest two-patch network. More precisely, the main theorem of the Connection Graph Method can be reformulated for the metacommunity (1)-(3) as follows.

Theorem. Synchronization in the network (1)-(3) with a given dispersal profile $H$ is globally asymptotically stable, i.e. it arises from any initial conditions if the dispersal $d$ exceeds the critical value $d^{*}$, where

$$
d^{*}=\frac{2 d_{12}^{*}}{n} \cdot \max _{k} z_{k} \quad \text { for } k=1, \ldots, m
$$

Here, $d_{12}^{*}$ is the dispersal sufficient for global synchronization of two patches with the same dispersal profile $H, m$ is the number of edges on the graph, and $n$ is the number of patches. The quantity $z_{k}=\sum_{j>i ; k \in P_{i j}}^{n}\left|P_{i j}\right|$ is the sum of the lengths of all chosen paths $P_{i j}$ which pass through a given edge $k$ that belongs to the connection graph.

The first step of the method is to find the critical dispersal $d_{12}^{*}$ for two mutually coupled patches. Analytical derivation of an upper bound $d_{12}^{*}$ for the global stability 


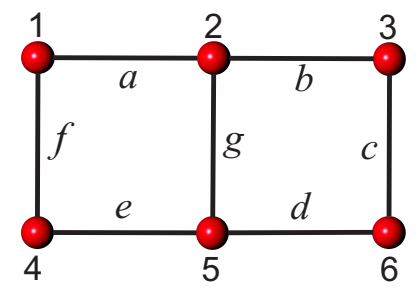

Figure 7. Example of a network configuration under consideration.

of the synchronous state of the reference model (3) with $\delta=0$ in the case of two patches with dispersal profile $H^{0}=\operatorname{diag}[1,1,1]$ is given in Appendix. The proof can also be extended to the case of dispersal profile $H^{\mathrm{II}}=\operatorname{diag}[0,1,0]$.

It is worth noticing that the analysis of synchronization given in Appendix also supports the statement made in the previous section regarding the role of the stabilizing factors in synchronization. That is, it shows that the stabilizing factors introduced to the reference model $(\delta \neq 0)$ also favor global synchronization. The details are also given in Appendix.

The second step is to calculate the factor $z_{k}$ which does not depend on the individual patch dynamics and is determined by the connection graph. This calculation is straightforward and performed as follows. We first choose a set of paths $\left\{P_{i j} \mid i, j=1, \ldots, n, j>i\right\}$ (typically, the shortest paths), one for each pair of patches $i, j$, and determine their lengths $\left|P_{i j}\right|$, the number of edges in each $P_{i j}$. Then, for each edge $k$ of the connection graph we calculate the sum $z_{k}$ of the lengths of all $P_{i j}$ passing through $k$. Finally, we determine the edge $k$ with the maximum $z_{k}$ such that the sum of the lengths of all paths through $k$ is maximal. This is the link having the maximum traffic load on it.

The number of possible choices of paths is normally huge. However, most of these choices are clearly suboptimal. Usually, one takes for $P_{i j}$ the shortest path from patch $i$ to patch $j$. Sometimes, however, a different choice of paths can lead to smaller lower bounds [5].

We shall now illustrate our criterion by applying it to a typical metapopulation network, depicted in Fig. 7. Consider the network (1)-(3) with the connection graph shown in Fig. 1 and dispersal profile $H^{0}=\operatorname{diag}[1,1,1]$. As $d_{12}^{*}$ for this network is already calculated (see Appendix), we only need to calculate $z_{k}$ to establish the upper bound for global synchronization in the network.

Choose first the paths between the nodes. Our choice is $P_{12}=a, P_{13}=a b$, $P_{14}=f, P_{15}=a g, P_{16}=$ fed, $P_{23}=b, P_{24}=a f, P_{25}=g, P_{26}=b c, P_{34}=c d e$, $P_{35}=b g, P_{36}=c, P_{45}=e, P_{46}=e d, P_{56}=d$. Calculate the sum of path lengths passing through edge

$$
\begin{aligned}
& a: z_{a}=\left|P_{12}\right|+\left|P_{13}\right|+\left|P_{15}\right|+\left|P_{24}\right|=1+2+2+2=7 \\
& b: z_{b}=\left|P_{13}\right|+\left|P_{23}\right|+\left|P_{26}\right|+\left|P_{35}\right|=2+1+2+2=7 \\
& c: z_{c}=\left|P_{26}\right|+\left|P_{34}\right|+\left|P_{36}\right|=2+3+1=6 \\
& d: z_{d}=\left|P_{16}\right|+\left|P_{34}\right|+\left|P_{46}\right|+\left|P_{56}\right|=3+3+2+1=9 \\
& e: z_{e}=\left|P_{16}\right|+\left|P_{34}\right|+\left|P_{45}\right|+\left|P_{46}\right|=3+3+1+2=9 \\
& f: z_{f}=\left|P_{14}\right|+\left|P_{16}\right|+\left|P_{24}\right|=1+3+2=6 \\
& g: z_{g}=\left|P_{15}\right|+\left|P_{25}\right|+\mid P_{35}=2+1+2=5
\end{aligned}
$$

and take the maximum $z_{d}=z_{e}=9$ as an upper bound. Thus, the upper bound (9) for global synchronization becomes

$$
d^{*}=\frac{2 d_{12}^{*}}{6} \cdot 9=3 d_{12}^{*}
$$


where $d_{12}^{*}$ is given by $(26)$.

Calculations of $\max z_{k}$ for various network configurations can be found in [4-7].

One should also remark that the method for proving global synchronization is valid for networks of slightly nonidentical patches(up to $10-15 \%$ parameter mismatch.) In this case, perfect synchronization cannot exist anymore, but approximate synchronization is still possible and therefore similar global stability conditions of approximate synchronization can be derived by means of the presented method and the technique developed in [4].

\section{Concluding remarks}

...

The stability analysis of synchronization discussed in the paper can also be applied to metapopulations with directed connection graphs, under the constraint that the graph allows synchronization of all the patches. Indeed, synchrony in directly coupled networks is only possible if there is at least one patch which directly or indirectly influences all the others. In terms of the connection graph, this amounts to the existence of a uniformly directed tree involving all the vertices. A star-coupled network where secondary nodes drive the hub is a counter example, where such a tree does not exist and synchronization is impossible. The generalization of the connection graph method to arbitrary asymmetric coupling is reported in [7].

- Novelty and importance of the results

- Possible extensions (clusters $+\ldots$ )

\section{Acknowledgements}

Financial support was provided by Landau Network-Centro Volta and by the Italian Ministry of the University under grant PRIN 2005098133.

\section{Appendix}

In this appendix, we prove global stability of synchronization in the simplest twopatch network (1) and derive an upper bound for the synchronization threshold. For the sake of clarity, we choose the dispersion profile to be $H=\operatorname{diag}[1,1,1]$ such that we assume that the resource, consumers, and predators disperse with equal dispersal $d$. Generalization of the proof to the case of heterogeneous dispersal coefficients is straightforward.

Before having started the study of global stability of the synchronization solution, we first need to prove the eventual dissipativeness of system (1). That is, we need to show that there exists a region (an absorbing domain $A$ ) which attracts all trajectories of the system from the outside, and there are no trajectories which go to infinity.

\section{A1. Eventual dissipativeness}

To get the lower bound for trajectories of the isolated patch system (3), it is sufficient to observe that $\dot{x}=0$ at the point $x=0, \dot{y}=0$ at $y=0$, and $\dot{z}=0$ at $z=0$. Therefore, trajectories may not leave the region $A^{+}=\{x>0, y>0, z>0\}$. This makes a clear sense as the population sizes expressed by $x, y, z$ may not be negative. 
To make our calculation more transparent, let us plug the values $e_{1}=1$ and $e_{2}=1$ from the parameter set (8) into system (3), and thus transform (3) into the system

$$
\begin{aligned}
& \dot{x}=r x\left(1-\frac{x}{K}\right)-\frac{a_{1} x y}{1+a_{1} b_{1} x}, \\
& \dot{y}=\frac{a_{1} x y}{1+a_{1} b_{1} x}-m_{1} y-\frac{a_{2} y z}{1+a_{2} b_{2} y} \\
& \dot{z}=\frac{a_{2} y z}{1+a_{2} b_{2} y}-m_{2} z
\end{aligned}
$$

Below we derive an upper bound for the absorbing domain of (10).

Consider a family of planes

$$
V=x+y+z-c
$$

where $c \geq c^{*}$ is to be determined.

Our goal is to find the constant $c^{*}$ that makes $V$ a Liapunov-type function such that its constant levels become surfaces without contact for system (10), i.e. the vector field of (10) on these surfaces is oriented toward the origin.

The derivative of $V$ with respect to system (10) is calculated as follows

$$
\dot{V}=\dot{x}+\dot{y}+\dot{z}=r x\left(1-\frac{x}{K}\right)-m_{1} y-m_{2} z,
$$

where the terms $\pm \frac{a_{1} x y}{1+a_{1} b_{1} x}$ and $\pm \frac{a_{2} y z}{1+a_{2} b_{2} y}$ have cancelled out.

Since $m_{2}<m_{1}$ (0.0037 vs. 0.4), we can bound the right hand side (RHS) of (12) by replacing the term $m_{1} y$ with $m_{2} y$. This yields

$$
\dot{V}<r x\left(1-\frac{x}{K}\right)-m_{2}(y+z)<\left[r x-\frac{r x^{2}}{K}-m_{2}(c-x)\right]_{V=0}
$$

Here, we have replaced the term $m_{2}(y+z)$ with its minimum value $m_{2}(c-x)$ it reaches when $V=x+y+z-c=0$ and, hence, $y+z=c-x$.

Therefore, the derivative $\dot{V}$ is negative along trajectories of (10) if $r x-\frac{r x^{2}}{K}-$ $m_{2}(c-x)<0$. Let's find the values of $c$ that make this inequality true.

The roots of the equation

$$
-\frac{r x^{2}}{K}+\left(r+m_{2}\right) x-m_{2} c=0
$$

are $x_{1,2}=\frac{\left(r+m_{2}\right) \pm \sqrt{\left(r+m_{2}\right)^{2}-4 m_{2} c r / K}}{2 r / K}$. Equation (14) has no solutions, and therefore $\dot{V}$ is always negative, if $c>c^{*}=\frac{K}{4 m_{2} r}\left(r+m_{2}\right)^{2}$. Thus

$$
c^{*}=\frac{K}{4 m_{2} r}\left(r+m_{2}\right)^{2}
$$

is the critical level of $V$ such that $\dot{V}<0$ when $V>c^{*}$ and therefore $V=c^{*}$ is an upper bound for all trajectories of (10).

Combining this bound with the domain $A^{+}$we arrive at the following absorbing domain $A=A^{+}\{x>0, y>0, z>0\} \cap\left\{V<c^{*}\right\}$. Hence, the variables of (10) are 
bounded as follows:

$$
x<c^{*}, y<c^{*},, z<c^{*},
$$

where $c^{*}$ is defined in (15).

Similar to [8], it can be easily shown that the bound (16) is valid for the variables of each subsystem (patch) (10) of network (1).

\section{A2. Proof of global synchronization}

Consider the following two-patch network (1) of subsystems (10):

$$
\left\{\begin{array}{l}
\dot{x}^{(1)}=f\left(x^{(1)}\right)-g\left(x^{(1)}\right) y^{(1)}+d\left(x^{(2)}-x^{(1)}\right) \\
\dot{y}^{(1)}=g\left(x^{(1)}\right) y^{(1)}-m_{1} y^{(1)}-\tilde{g}\left(y^{(1)}\right) z^{(1)}+d\left(y^{(2)}-y^{(1)}\right), \\
\dot{z}^{(1)}=\tilde{g}\left(y^{(1)}\right) z^{(1)}-m_{2} z^{(1)}+d\left(z^{(2)}-z^{(1)}\right) \\
\dot{x}^{(2)}=f\left(x^{(2)}\right)-g\left(x^{(2)}\right) y^{(2)}+d\left(x^{(1)}-x^{(2)}\right) \\
\dot{y}^{(2)}=g\left(x^{(2)}\right) y^{(2)}-m_{1} y^{(2)}-\tilde{g}\left(y^{(2)}\right) z^{(2)}+d\left(y^{(1)}-y^{(2)}\right), \\
\dot{z}^{(2)}=\tilde{g}\left(y^{(2)}\right) z^{(2)}-m_{2} z^{(2)}+d\left(z^{(1)}-z^{(2)}\right)
\end{array}\right.
$$

where for simplicity we have used the notations $f\left(x^{(i)}\right)=r x^{(i)}\left(1-\frac{x^{(i)}}{K}\right), g\left(x^{(i)}\right) \equiv$ $g\left(x^{(i)}, a_{1}, b_{1}\right)=\frac{a_{1} x^{(i)}}{1+a_{1} b_{1} x^{(i)}}$ and $\tilde{g}\left(y^{(i)}\right) \equiv g\left(y^{(i)}, a_{2}, b_{2}\right)=\frac{a_{2} y^{(i)}}{1+a_{2} b_{2} y^{(i)}}$ for $i=1,2$.

Introducing the notations for the differences and sums

$$
\begin{array}{ll}
X=\frac{x^{(2)}-x^{(1)}}{2} & u=\frac{x^{(1)}+x^{(2)}}{2} \\
Y=\frac{y^{(2)}-y^{(1)}}{2} & v=\frac{y^{(1)}+y^{(2)}}{2} \\
Z=\frac{z^{(2)}-z^{(1)}}{2} & w=\frac{z^{(1)}+z^{(2)}}{2}
\end{array}
$$

we obtain the difference equation system

$$
\left\{\begin{array}{l}
\dot{X}=\frac{1}{2}\left\{\left[f\left(x^{(2)}\right)-f\left(x^{(1)}\right)\right]-\left[g\left(x^{(2)}\right) y^{(2)}-g\left(x^{(1)}\right) y^{(1)}\right]\right\}-2 d X \\
\dot{Y}=\frac{1}{2}\left\{\left[g\left(x^{(2)}\right) y^{(2)}-g\left(x^{(1)}\right) y^{(1)}\right]-\left[\tilde{g}\left(y^{(2)}\right) z^{(2)}-\tilde{g}\left(y^{(1)}\right) z^{(1)}\right]\right\}-\left(m_{1}+2 d\right) Y \\
\dot{Z}=\frac{1}{2}\left\{\left[\tilde{g}\left(y^{(2)}\right) z^{(2)}-\tilde{g}\left(y^{(1)}\right) z^{(1)}\right]\right\}-\left(m_{2}+2 d\right) Z
\end{array}\right.
$$

To get rid of the variables $\left.\left.\left.x^{(1)}\right), y^{(1)}\right), z^{(1)}\right)$ and $\left.\left.\left.x^{(2)}\right), y^{(2)}\right), z^{(2)}\right)$ in (19), we apply the Mean Value Theorem to the differences of the functions such that $\left[f\left(x^{(2)}\right)-\right.$ $f\left(x^{(1)}\right)=f_{x}\left(\xi_{1}\right)\left(x_{2}-x_{1}\right)=2 f_{x}\left(\xi_{1}\right) X$, where $\left.\left.\xi_{1} \in\left[x^{(1)}\right), x^{(2)}\right)\right] ;\left[g\left(x^{(2)}\right)-g\left(x^{(1)}\right)=\right.$ $g_{x}\left(\xi_{2}\right)\left(x_{2}-x_{1}\right)=2 g_{x}\left(\xi_{2}\right) X$, where $\left.\left.\xi_{2} \in\left[x^{(1)}\right), x^{(2)}\right)\right]$. At the same time, it follows from (18) that

$$
\begin{aligned}
& x^{(2)}=u+X, \mathrm{x}^{(1)}=u-X, \\
& y^{(2)}=v+Y, \quad y^{(1)}=v-Y, \\
& z^{(2)}=w+Z, \quad \mathrm{z}^{(1)}=w-Z,
\end{aligned}
$$

such that the differences of other functions can be transformed as follows $\left[g\left(x^{(2)}\right) y^{(2)}-g\left(x^{(1)}\right) y^{(1)}\right]=\left[g\left(x^{(2)}\right)(v+Y)-g\left(x^{(1)}\right)(v-Y)\right]=$ $\left[g\left(x^{(2)}\right)-g\left(x^{(1)}\right)\right] v+[g(u+X)+g(u-X)] Y=2 g_{x}\left(\xi_{2}\right) X v+$ $[g(u+X)+g(u-X)] Y$, and similarly $\left[\tilde{g}\left(y^{(2)}\right) z^{(2)}-\tilde{g}\left(y^{(1)}\right) z^{(1)}\right]=2 \tilde{g}_{y}(\eta) Y w+$ $[\tilde{g}(v+Y)+g(v-Y)] Z$, where $\left.\left.\eta \in\left[y^{(1)}\right), y^{(2)}\right)\right]$. 
Thus, difference system (19) simplifies to

$$
\left\{\begin{aligned}
\dot{X}= & {\left[f_{x}\left(\xi_{1}\right)-g_{x}\left(\xi_{2}\right) v-2 d\right] X-\frac{1}{2}[g(u+X)+g(u-X)] Y, } \\
\dot{Y}= & g_{x}\left(\xi_{2}\right) v X+\left\{\frac{1}{2}[g(u+X)+g(u-X)]-\tilde{g}_{y}(\eta) w-\left(m_{1}+2 d\right)\right\} Y- \\
& \frac{1}{2}[\tilde{g}(v+Y)+g(v-Y)] Z \\
\dot{Z}= & \tilde{g}_{y}(\eta) w Y+\left\{\frac{1}{2}[\tilde{g}(v+Y)+g(v-Y)]-\left(m_{2}+2 d\right)\right\} Z
\end{aligned}\right.
$$

Our goal is to obtain conditions under which the coupled system (21) is convergent and its trivial equilibrium $\{X=0, Y=0, Z=0\}$, corresponding to the synchronous solution $\left\{x^{(1)}=x^{(2)}, y^{(1)}=y^{(2)}, z^{(1)}=z^{(2)}\right\}$ is globally asymptotically stable.

The proof that the origin can be globally stable involves the construction of a Lyapunov function, a smooth, positive definite function that decreases along trajectories of system (21).

Consider the Lyapunov function

$$
\Phi=\frac{1}{2}\left(X^{2}+Y^{2}+Z^{2}\right) .
$$

Calculating the time derivative of $\Phi$ with respect to system (21), we obtain

$$
\dot{\Phi}=-\left[A_{11} X^{2}+2 A_{12} X Y+A_{22} Y^{2}+2 A_{23} Y Z+A_{33} Z^{2}\right],
$$

where

$$
\begin{aligned}
& A_{11}=2 d-f_{x}\left(\xi_{1}\right)+g_{x}\left(\xi_{2}\right) V \\
& A_{12}=\frac{1}{4}[g(u+X)+g(u-X)]-\frac{1}{2} g_{x}\left(\xi_{2}\right) V, \\
& A_{22}=2 d+m_{1}+\tilde{g}_{y}(\eta) w-\frac{1}{2}[g(u+X)+g(u-X)], \\
& A_{23}=\frac{1}{4}[\tilde{g}(u+X)+\tilde{g}(u-X)]-\frac{1}{2} \tilde{g}_{y}(\eta) w, \\
& A_{33}=2 d+m_{2}-\frac{1}{2}[\tilde{g}(v+Y)+g(v-Y)] .
\end{aligned}
$$

The derivative $\dot{\Phi}$ is negative along trajectories of system (21) if the quadratic form $S=A_{11} X^{2}+2 A_{12} X Y+A_{22} Y^{2}+2 A_{23} Y Z+A_{33} Z^{2}$ is positive definite. To make the proof simpler, we can split the quadratic form $S$ as follows: $S=S_{1}+S_{2}$, where $S_{1}=A_{11} X^{2}+2 A_{12} X Y+A_{22} Y^{2} / 2$ and $S_{2}=A_{22} Y^{2} / 2+2 A_{23} Y Z+A_{33} Z^{2}$. Here, the coefficients $A_{11}, A_{22}$, and $A_{33}$ depend on the coupling strength $d$ and favor positiveness of the two quadratic forms $S_{1}$ and $S_{2}$. For $S_{1}$ and $S_{2}$ to be positive, $A_{11}, A_{22}$, and $A_{33}$ must overcome the negative contribution of the coefficients $A_{23}$ and $A_{33}$. This is achieved when the coupling strength $d$ exceeds a critical value.

Applying the Sylvester criterion for positive definiteness of the two quadratic forms, we obtain the conditions

$$
\begin{aligned}
& A_{11}>0, A_{22}>0, A_{33}>0, \\
& \frac{1}{2} A_{11} A_{22}>A_{12}^{2}, \frac{1}{2} A_{22} A_{33}>A_{23}^{2} .
\end{aligned}
$$

The functions that are present in the A's coefficients can be bounded as follows

$$
\begin{gathered}
f_{x}\left(\xi_{1}\right) \leq f_{x}(0) \equiv r, \quad g_{x}\left(\xi_{2}\right) V \geq 0, \tilde{g}_{y}(\eta) w \geq 0 \\
g(u+X)+g(u-X) \leq 2 g\left(c^{*}\right)<\frac{2}{b_{1}} \\
\tilde{g}(u+X)+\tilde{g}(u-X) \leq 2 \tilde{g}(c *)<\frac{2}{b_{2}} \\
\tilde{g}(u+X)+\tilde{g}(u-X) \leq 2 \tilde{g}(c *)<\frac{2}{b_{2}} \\
\tilde{g}(v+Y)+g(v-Y) \leq 2 \tilde{g}(c *)<\frac{2}{b_{2}} .
\end{gathered}
$$


Here, we have bounded from above the arguments of functions $g$ and $\tilde{g}$ by constant $c^{*}$ (16) that is the upper bound for $x-, y-$, and $z$-trajectories of system (17).

Using bounds (25), we can make conditions (24) more conservative by minimizing the favorable terms $A_{11}, A_{22}, A_{33}$ and maximizing $A_{12}, A_{23}$. This yields the following bounds

$$
\begin{gathered}
d>r / 2 ; \quad d>\frac{1}{2}\left[\frac{1}{b_{1}}-m_{1}\right] ; \quad d>\frac{1}{2}\left[\frac{1}{b_{2}}-m_{2}\right] \\
(2 d-r)\left(2 d+m_{1}-1 / b_{1}\right)>\frac{1}{2 b_{1}^{2}} \\
\left(2 d+m_{1}-1 / b_{1}\right)\left(2 d+m_{2}-1 / b_{2}\right)>\frac{1}{2 b_{2}^{2}}
\end{gathered}
$$

For the given set of parameters $(3)$ where $(2 d-r)>\left(2 d+m_{1}-1 / b_{1}\right),\left(2 d+m_{1}-\right.$ $\left.1 / b_{1}\right)<\left(2 d+m_{2}-1 / b_{2}\right)$, and $1 / b_{1}^{2}>1 / b_{2}^{2}$ we can replace the last two equalities in $(26)$ by

$$
d>d_{12}^{*}=\left(\frac{\sqrt{2}}{2 b_{1}}-m_{1}+1 / b_{1}\right) / 2
$$

This inequality serves as an upper bound for the inequalities (26). Thus, it gives a bound for the coupling strength $d$, sufficient to make the quadratic form $\dot{\Phi}$ negative definite. This, in turn, guarantees global stability of synchronization in the coupled system (17) with individual system's parameters from the set (3). This completes the proof.

Note that a similar stability approach to global synchronization is also applicable to a two-patch network of modified systems (3) with $\delta \neq 0$. In this case, the difference system (19) will have extra terms. An important observation is that the stabilizing factors $1,3,4$ and 6 contribute to system (19) the terms that favor the stability of the origin, and thus aim at stabilizing the synchronous solution. For example, the addition of factor 1 to the two patches yields an extra term $-2 \delta v Y$ to the $Y$ equation of the difference system (19). Note that $v=\frac{y^{(1)}+y^{(2)}}{2}$ is always nonnegative, therefore the term $-2 \delta v Y$ makes the trivial equilibrium of system (19) more stable and therefore lowers the critical value of the dispersal required for global synchronization. The same stability arguments carry over to other stabilizing factors 3,4 and 6 . Thus, our stability discussions on the role of the stabilizing factors in global synchronization are consistent with the claims made in section 3 .

\section{References}

99

[1] M. Barahona and L.M. Pecora, Synchronization in small-world systems, Physical Review Letters 89 (2002), 054101.

[2] J.R. Beddington, Mutual interference between parasites or predators and its effect on searching effciency, Journal of Animal Ecology 44 (1975), pp. 331-340.

[3] V.N. Belykh, N.N. Verichev, L.J. Kocarev, and L.O. Chua, in Chua's Circuit: A Paradigm for Chaos, edited by R.N. Madan, World Scientific, Singapore, 1993, 325.

[4] V.N. Belykh, I. Belykh, and M. Hasler, Connection graph stability method for synchronized coupled chaotic systems, Physica D 195 (2004), pp. 159-187.

[5] I. Belykh et al., Synchronization and graph topology, International Journal of Bifurcation and Chaos 11 (2005), pp. 3423-3433. 
[6] I. Belykh, V. Belykh, and M. Hasler, Synchronization in asymmetrically coupled networks with node balance, Chaos 16 (2006) 015102.

[7] I. Belykh, V. Belykh, and M. Hasler, Generalized connection graph method for synchronization in asymmetrical networks, Physica D 224 (2006) pp. 4251.

[8] I. Belykh, V.N. Belykh, K.V. Nevidin, and M. Hasler, Persistent clusters in lattices of coupled nonidentical chaotic systems, Chaos 13 (2003) pp. 165-178.

[9] O.N. Bjørnstad, N.C. Stenseth, and T. Saitoh, Synchrony and scaling in dynamics of voles and mice in northern Japan, Ecology 80 (1999), pp. 622-637.

[10] B. Blasius, A. Huppert, and L. Stone, Complex dynamics and phase synchronization in spatially extended ecological systems, Nature 399 (1999) pp. 354-359.

[11] M.G. Bulmer, A statistical analysis of the 10-year cycle in Canada, Journal of Animal Ecology 43 (1974), pp. 701-718.

[12] I.M. Cattadori et al., Synchrony, scale and temporal dynamics of rock partridge (Alectoris graeca saxatilis) populations in the dolomites, Journal of Animal Ecology 68 (1999), pp. 540-549.

[13] B. Cazelles and G. Boudjema, The Moran effect and phase synchronization in complex spatial community dynamics, The American Naturalist 157 (2001), pp. 670-676.

[14] B. Cazelles, S. Bottani, and L. Stone, Unexpected coherence and conservation, Proceedings of the Royal Society of London B 268 (2001), pp. 2595-2602.

[15] A. Colombo, F. Dercole, and S. Rinaldi, Remarks on metacommunity synchronization with application to prey-predator systems, The American Naturalist (2008), in press.

[16] F. Dercole, D. Loiacono, and S. Rinaldi, Synchronization in ecological networks: A byproduct of Darwinian evolution?, International Journal of Bifurcation and Chaos 7 (2007), pp. 2435-2446.

[17] M. Doebeli and G.D. Ruxton, Evolution of dispersal rates in metapopulation models: Branching and cyclic dynamics in phenotype space, Evolution 51 (1997), pp. 1730-1741.

[18] C.S. Elton, Periodic fluctuations in the numbers of animals, British Journal of Experimental Biology 2 (1924), pp. 119-163.

[19] C.S. Elton and M. Nicholson, The ten-year cycle in numbers of the lynx in Canada, Journal of Animal Ecology 11 (1942), pp. 215-244

[20] B.T. Grenfell et al., Noise and determinism in synchronized sheep dynamics, Nature 394 (1998), pp. 674-677.

[21] I. Hanski and I.P. Woiwod, Spatial synchrony in the dynamics of moth and aphid populations, Journal of Animal Ecology 62 (1993), pp. 656-668.

[22] M.A. Harrison, Y.-C. Lai, R.D. Holt, Dynamical mechanism for coexistence of dispersing species, Journal of Theoretical Biology 213 (2001), pp. 53-72.

[23] A. Hastings and T. Powell, Chaos in a 3-species food-chain, Ecology 72 (1991), pp. 896-903.

[24] K. Higgins et al., Stochastic dynamics and deterministic skeletons: Population behavior of Dungeness crab, Science 276 (1997), pp. 1431-1435.

[25] R.A. Ims and H. Steen, Geographical synchrony in microtine rodent populations: A theoretical explanation of the role of nomadic avian predators, Oikos 57 (1990), pp. 381-387.

[26] V.A.A. Jansen and A.L. Lloyd, Local stability analysis of spatially homogeneous solutions of multipatch systems, Journal of Mathematical Biology 41 (2000), pp. 232-252.

[27] D.M. Johnson et al., Circumpolar variation in periodicity and synchrony among gypsy moth populations, Journal of Animal Ecology 74 (2005), pp. 882-892.

[28] A.A. King and W.M. Schaffer, The geometry of a population cycle: A mechanistic model of snowshoe hare demography, Ecology 82 (2001), pp. 814-830.

[29] W.D. Koenig, Spatial autocorrelation of ecological phenomena, Trends in Ecology Evolution 14 (1999), pp.22-26.

[30] — Spatial synchrony of monarch butterflies, American Midland Naturalist 155 (2006), pp.39-49.

[31] Y.A. Kuznetsov, O. De Feo, and S. Rinaldi, Belyakov homoclinic bifurcations in a tritrophic food chain model, SIAM Journal on Applied Mathematics 62 (2001), pp. 462-487.

[32] A.M. Liebhold, W.D. Koenig, and O.N. Bjørnstad, Spatial synchrony in population dynamics, Annual Review of Ecology, Evolution and Systematics 35 (2004), pp. 467-490.

[33] A.M. Liebhold, D.M. Johnson, and O.N. Bjørnstad, Geographic variation in density dependent dynamics impacts the synchronizing effect of dispersal and regional stochasticity, Population Ecology 48 (2006), pp. 131-138.

[34] A.L. Lloyd and R.M. May, Synchronicity, chaos and population cycles: Spatial coherence in an uncertain world, Trends in Ecology Evolution 14 (1999), pp. 417-418.

[35] S. Maggi and S. Rinaldi, Synchronization and peak-to-peak dynamics in networks of low-dimensional chaotic oscillators, International Journal of Bifurcation and Chaos 16 (2006), pp. 3631-3642.

[36] P.A.P. Moran, The statistical analysis of the Canadian lynx cycle. II. Synchronisation and meteorology, Australian Journal of Zoology 1 (1953), pp. 291-298. 
[37] J.H. Myers, Synchrony in outbreaks of forest lepidoptera: A possible example of the Moran effect, Ecology 79 (1998), pp.1111-1117.

[38] L.M. Pecora and T.L. Carroll, Master stability functions for synchronized coupled systems, Physical Review Letters 80 (1998), pp. 2109-2112.

[39] A.Yu. Pogromsky and H. Nijmeijer, IEEE Trans. Circuits Syst., I: Fundam. Theory Appl. 48 (2001), pp. 152-??.

[40] E. Post, Large-scale climate synchronizes the timing of flowering by multiple species, Ecology 84 (2003), pp. 277-281.

[41] E. Post and M.C. Forchhammer, Synchronization of animal population dynamics by large-scale climate, Nature 420 (2002), pp.168-171.

[42] K. Ramasubramanian and M.S. Sriram, A comparative study of computation of Lyapunov spectra with different algorithms, Physica D 139 (2000), pp. 72-86.

[43] E. Ranta et al., Synchrony in population dynamics, Proceedings of the Royal Society of London B 262 (1995), pp. 113-118.

[44] E. Ranta, J. Lindström, and H. Lindén, Synchrony in tetraonid population dynamics, Journal of Animal Ecology 64 (1995), pp. 756-776.

[45] E. Ranta et al., The Moran effect and synchrony in population dynamics, Oikos 78 (1997), pp. 136142.

[46] S. Rinaldi and A. Gragnani, Destabilizing factors in slow-fast systems, Ecological Modelling 180 (2004), pp. 445-460.

[47] M.L. Rosenzweig and R.H. MacArthur, Graphical representation and stability conditions of predatorprey interactions, The American Naturalist 97 (1963), pp. 209-223.

[48] T. Royama, Moran effect on nonlinear population processes, Ecological Monographs 75 (2005), pp. $277-293$.

[49] G.D. Ruxton, J.L. Gonzalez-Andujar, and J.N. Perry, Mortality during dispersal an the stability of a metapopulation, Journal of Theoretical Biology 186 (1997), pp. 389-396.

[50] M.K. Schwartz et al., DNA reveals high dispersal synchronizing the population dynamics of Canadian lynx, Nature 415 (2002), pp. 520-522.

[51] A.R.E. Sinclair et al., Can the solar cycle and climate synchronize the snowshoe hare cycle in Canada? Evidence from tree rings and ice cores, The American Naturalist 141 (1993), pp. 173-198.

[52] C.H. Smith, Spatial trends in Canadian snowshoe hare Lepus americanus population cycles, The Canadian Field-Naturalist 97 (1983), pp. 151-160.

[53] O.L. Sutclife, C.D. Thomas, and D. Moss, Spatial synchrony and asynchrony in butterfly populations dynamics, Journal of Animal Ecology 65 (1996), pp.85-95.

[54] C.W. Wu and L.O. Chua, IEEE Trans. Circuits Syst., I: Fundam. Theory Appl. 43 (1996), pp. 161-??.

[55] C.W. Wu, Synchronization in Coupled Chaotic Circuits and Systems, edited by L.O. Chua, World Scientific Series on Nonlinear Science, Series A Vol. 41, World Scientific, Singapore, 2002. 